\title{
Impact of Acceptance and Commitment Therapy on Valuing Behaviour of Parents of Children with Neurodevelopmental Disorders
}

\author{
Shuvabrata Poddar ${ }^{1}$, Vinod K Sinha ${ }^{2}$ and Urbi Mukherjee ${ }^{3}$ \\ ${ }^{1}$ Department of Applied Psychology, Kazi Nazrul University, India \\ ${ }^{2}$ Department of Psychiatry, Central Institute of Psychiatry, India \\ ${ }^{3}$ Department of Psychology, Dr. A.P.J. Abdul Kalam Government College, India
}

Submission: April 11, 2017; Published: April 24, 2017

*Corresponding author: Shuvabrata Poddar, Assistant Professor and Coordinator, Department of Applied Psychology, Kazi Nazrul University, India, Email: shuvabrata.poddar@gmail.com

\begin{abstract}
Background: Acceptance and Commitment Therapy (ACT) focuses on accepting things that are beyond control and on commitment to possibly changeable aspects by increasing the psychological flexibility of the person, thereby aiding to better realistic adjustments.

Objectives: The present study aims at studying the effectiveness of ACT on parents having their children with neurodevelopmental disorders, who inevitably face enormous challenges by the developmental deficits of their offspring.

Methods: The study followed hospital based repeated measures study design. Sample consisted 5 parents of children with neurodevelopmental disorders receiving intervention. The present study used a 9-session protocol of ACT spread over 6 weeks. Assessment measures were taken for values and psychological flexibility. Prior to the treatment, baseline measures were taken and further compared with post-treatment and follow up measures using appropriate statistical analyses.
\end{abstract}

Results: Pre- to post- treatment improvements were evident in values and psychological flexibility.

Conclusions: Findings implied that ACT's effectiveness on parents having children diagnosed with neurodevelopmental disorders. The mechanism of change reflected in the study, is highly consistent with the theory and philosophy behind ACT and underscored the need for longer treatment.

Keywords: Acceptance and Commitment Therapy; Parents; Neurodevelopmental Disorders; Values

Abbreviations: ACT: Acceptance and Commitment Therapy; DSM-5: Diagnostic and Statistical Manual of Mental Disorders- Fifth edition; ASD: Autism Spectrum Disorders; NDs: Neurodevelopmental Disorders; BPT: Behavioural Parent Training; MBSR: Mindfulness Based Stress Reduction; SDCSP: Socio-Demographic and Clinical Data Sheet; AAQ: Acceptance and Action Questionnaire; PVQ: Personal Values Questionnaire; AFQ: Acceptance and Fusion Questionnaire

\section{Introduction}

In the recent nosology of Diagnostic and Statistical Manual of Mental Disorders, fifth edition (DSM-5), the Autism spectrum disorders (ASD) are categorized under Neurodevelopmental disorders (NDs) that refer to a group of conditions that typically manifest early in development and are characterised by developmental deficits that produce impairments of personal, social, academic or occupational functioning. The range of developmental deficits varies from specific limitations to global impairments and NDs frequently co-occur. ASD is characterized by persistent deficits in social communication and reciprocal social interactions across multiple contexts and restricted, repetitive patterns of behaviour, interests and activities.

When parents first encounter such debilitating condition of their child/ children and come to know about the irreversible status of the problem, their immediate reaction would become overwhelmed by indecisiveness and confusion as such problems 
of their children would mallet their expectation of being a parent which is potential enough to create parenting stress-an aversive psychological reaction to the demands of being a parent [1]. They tend to feel responsible and blamed for their children's conditions, guilty and ashamed, and feel even hatred, anger, and blame toward their partners for their perceived responsibility [2]. They experience high levels of chronic stress [3], especially owing to the child's need for unique nurturance which is often inconvenient to them, specifically considering the socio-economic scenario and mental health awareness of our country, and also owing to the social limitations and constraints imposed from the poorly controlled behavioural manifestations of their children. These parents tend to have high rates of depressive and anxiety disorders [4] that negatively intermingles with psychosocial adjustment. Mothers in particular report difficulties with the impact on their lives of the child's condition [5]. Both parents tend to be exhausted and pessimistic about the future. All these clearly indicate the importance of implementing intervention for stressed parents having children with ASD.

As many of these children's problems are unlikely to change, at least quickly, acceptance of the "non-normative" conditions imposed by the pathology rather than challenging it becomes inevitable for the parents having children with ASD. Acceptance and Commitment Therapy [6,7] seems well- suited to address the issue as there are evidences that even widely used interventions through Behavioural Parent Training (BPT) overlook parents' internal experiences, their ability to learn and implement behavioural parenting techniques [8] and appropriately utilize those in changing contexts [9]. Moreover, the handful of attempts to address the needs of parents having children with ASD [10] are methodologically weak. However, preliminary studies have supported the effectiveness of mindfulness -based interventions, including mindfulness based stress reduction (MBSR) and an integrative programme combining BPT with mindfulness and narrative restructuring therapy.

ACT utilizes an eclectic mix of metaphor, paradox, and mindfulness skills, along with a wide range of experiential exercises and values-guided behavioural intervention that aim to undermine the power of Experiential Avoidance and Cognitive Fusion. This is based on two key themes: learning how to accept those things that are beyond control, and commit to changing those things that can be changed to make client's life better. The ACT practitioner targets six sub-processes in order to build psychological flexibility using two main components: acceptance and mindfulness processes (acceptance, defusion, the present moment, and a transcendent sense of self), and commitment and behavioral change processes (values, committed action, the present moment and a transcendent sense of self).

Values have been defined as chosen qualities of purposive action that can never be obtained as an object but can be instantiated moment by moment [11]. Central to this definition is the phrase -can never be obtained, which distinguishes values from goals. Values are meaningful life directions, not destinations. Values provide a rationale for participating in therapy at all, as acceptance, cognitive defusion, contact with the present moment, self-as-context, and committed action are all implemented in the service of a client's valued direction. For example, parents may value rich and meaningful relationships with their children. Having rich and meaningful relationships is something that doesn't end, as would a goal. ACT clinicians often prefer to talk about valuing, the behaviour, rather than values as an abstract concept. At the theoretical level, valuing is verbally constructing - globally desired life consequences [7] and at a practical level, valuing is choosing, moment-to-moment, to act consistently with that which provides meaning and vitality. Much of the research involving this particular component of the hexaflex (values) revolves around finding ways to measure it [12]. However, one study [13] examined the additive effect of a valuing component to an acceptance-based pain coping protocol which indicated that the addition of a values component led to significantly greater pain tolerance than acceptance alone, and both conditions led to greater pain tolerance than the control condition.

As there has been dearth of studies regarding intervention of parental distress in this line followed in this present study, it would abet to investigate relevant but comparatively unexplored arena in the management of caregivers' burden. Specifically, this study is an attempt to see whether increasing psychological flexibility in parents through acceptance and commitment therapy can lead to greater acceptance of their children's illness and whether there would be any change in the valuing behaviour of the parents.

\section{Material And Methods}

\section{Participants}

The sample comprised five parents ( 2 fathers and 3 mothers, having continuous contact with their children) of children and adolescents suffering from neurodevelopmental disorders. They were chosen from outpatient and inpatient services of the institute through purposive sampling method. The mean age of children was $11.6 \pm 3.57$ years. Mean age of the parents was $37.60 \pm 7.76$ years and their mean years of education were 12.00 \pm 3.00 years.

\section{Research Design}

The study followed a hospital-based before-after study design, categorizing its variables under two dimensions: outcome variables- valuing behaviour; process measure- psychological flexibility.

\section{Measures}

Socio-Demographic and Clinical Data Sheet (SDCS) (developed for the study): This data sheet used to obtain information about age, gender, education, duration of illness of their children, symptoms and treatment history. 
Acceptance and Action Questionnaire (AAQ), [14]: The Acceptance and Action Questionnaire (AAQ) is a 22-item questionnaire that measures psychological flexibility, the target process of ACT. Questions are rated on a 7-point Likert scale. Lower scores reflect greater psychological flexibility. The AAQ has adequate internal consistency $(\alpha=.70)$ and test-retest reliability $(\mathrm{r}=.64)$, and has demonstrated relationships with other psychopathology [14].

Personal Values Questionnaire (PVQ): [15] This questionnaire is used to identify values origin (intrinsic versus extrinsic), importance and commitment. It assesses nine value domains family relationships, friendships/social relationships, couples/romantic relationships, work/ career, educationschooling/personal growth and development, recreation/ leisure/sport, spirituality/religion, community/citizenship and health/physical well being. Every value domain has nine statements which are rated on a 5-point scale. This tool has been reported to have good psychometric properties.

\section{Procedure}

Participants meeting inclusion criteria for the study were assessed along state anxiety, depression, psychological flexibility and values by using Acceptance and Action Questionnaire (AAQ) and Personal Values Questionnaire respectively. Prior to the treatment, baseline measures were taken. Therapeutic program consisted 9 sessions spread over 6 weeks. Initial sessions (1-6) focused on understanding and acceptance of the disorder and Table 2a: Showing the comparison of the change of scores in various dom

\begin{tabular}{|c|c|c|c|c|c|c|}
\hline \multirow{2}{*}{$\begin{array}{l}\text { Personal Values } \\
\text { Questionnaire } \\
\text { (Importance) }\end{array}$} & \multicolumn{2}{|c|}{ Pre-Assessment(X) } & \multicolumn{2}{|c|}{ Post-Assessment(Y) } & \multirow{2}{*}{\multicolumn{2}{|c|}{ Wilcoxon signed rank test $\chi 2$}} \\
\hline & Mean \pm S.D & Mean Rank & Mean \pm S.D & Mean Rank & & \\
\hline Family & $14.20 \pm 3.19$ & 1.90 & $13.00 \pm 3.74$ & 1.80 & .77 & 0.67 \\
\hline Friends & $12.00 \pm 4.18$ & 2.20 & $11.60 \pm 2.88$ & 1.80 & .44 & 0.80 \\
\hline Couple & $13.20 \pm 4.43$ & 1.50 & $16.60 \pm 3.13$ & 2.40 & 2.80 & 0.24 \\
\hline Work & $11.60 \pm 3.64$ & 1.60 & $13.40 \pm 4.72$ & 2.20 & 1.50 & 0.47 \\
\hline Education & $12.80 \pm 2.77$ & 2.00 & $14.00 \pm 4.74$ & 2.10 & .11 & 0.94 \\
\hline Recreation & $10.20 \pm 3.76$ & 1.80 & $13.00 \pm 3.87$ & 2.50 & 2.77 & 0.24 \\
\hline Spirituality & $14.60 \pm 2.96$ & 2.10 & $14.00 \pm 5.24$ & 1.90 & .10 & 0.94 \\
\hline Community & $12.40 \pm 3.84$ & 1.70 & $12.60 \pm 3.84$ & 1.90 & 1.44 & 0.48 \\
\hline Health & $13.20 \pm 2.58$ & 2.10 & $13.40 \pm 4.21$ & 2.10 & .35 & 0.83 \\
\hline
\end{tabular}

*indicates significance at 0.05 level

Table $2 \mathbf{b}$ : showing the comparison of the change of scores in various domains of commitment towards personal values to personal values from pre to post.

\begin{tabular}{|c|c|c|c|c|c|}
\hline \multirow{2}{*}{$\begin{array}{c}\text { Personal Values } \\
\text { Questionnaire } \\
\text { (Importance) }\end{array}$} & \multicolumn{2}{|c|}{ Pre-Assessment(X) } & \multicolumn{2}{c|}{ Post-Assessment(Y) } \\
\cline { 2 - 6 } & Mean \pm S.D & Mean Rank & Mean \pm S.D & \multicolumn{2}{c|}{ Mean Rank } \\
\hline Family & $14.20 \pm 3.19$ & 1.90 & $13.00 \pm 3.74$ & 1.80 & .77 \\
\hline Friends & $12.00 \pm 4.18$ & 2.20 & $11.60 \pm 2.88$ & 1.80 & .44 \\
\hline Couple & $13.20 \pm 4.43$ & 1.50 & $16.60 \pm 3.13$ & 2.40 & 0.67 \\
\hline Work & $11.60 \pm 3.64$ & 1.60 & $13.40 \pm 4.72$ & 2.20 & 0.24 \\
\hline
\end{tabular}




\section{Psychology and Behavioral Science International Journal}

\begin{tabular}{|c|c|c|c|c|c|c|}
\hline Education & $12.80 \pm 2.77$ & 2.00 & $14.00 \pm 4.74$ & 2.10 & .11 & 0.94 \\
\hline Recreation & $10.20 \pm 3.76$ & 1.80 & $13.00 \pm 3.87$ & 2.50 & 2.77 & 0.24 \\
\hline Spirituality & $14.60 \pm 2.96$ & 2.10 & $14.00 \pm 5.24$ & 1.90 & .10 & 0.94 \\
\hline Community & $12.40 \pm 3.84$ & 1.70 & $12.60 \pm 3.84$ & 1.90 & 1.44 & 0.48 \\
\hline Health & $13.20 \pm 2.58$ & 2.10 & $13.40 \pm 4.21$ & 2.10 & .35 & 0.83 \\
\hline
\end{tabular}

*indicates significance at 0.05 level

Table-2a Shows that there is no significant change in the importance of different domains of values (Table $2 b$ ).

Table- $2 \mathrm{~b}$ Shows that the scores have changed significantly in commitment dimension of personal values in the domain of health from pre to post.

\section{Discussion}

Results indicated significant change in the process measure of psychological flexibility and in the health domain of commitment dimension of personal values from pre to post treatment, further indicating clear intervention effect along the selected aspects of outcome.

\section{Act and Psychological Flexibility}

It was hypothesized that AAQ scores would decrease between pre - test and post-test and the findings were consistent with the hypothesis. These findings suggest that, in general, avoidance and fusion decrease immediately in post -treatment. This is in direct contrast to the pattern of results found by Blackledge and Hayes [16]. In their study of ACT for parents of children diagnosed with autism, there were no significant changes in the Acceptance \& Action Questionnaire - 9 item (AAQ - 9) scores between pre -test and post- test, but there were significant changes from post- test to follow -up. The authors explained their findings in terms of an incubation effect, that is, that acceptance skills require time to be developed and practiced [16]. Other ACT studies have revealed similar effects, including an ACT intervention for smoking cessation [17] and ACT plus 12step facilitation for opiate addicts [14].

In the pilot study by Murrell et al. [17], acceptance/ experiential avoidance were as prominent in the treatment protocol as they were in the Blackledge and Hayes [1] study, yet none of the 3 parents evidenced reliable change in AAQ - II scores, with some scores actually increasing from pre-test to post -test. Murrell et al. [18] hypothesized that increased familiarity with the concepts of acceptance and experiential avoidance might be a confounding factor. That is, prior to treatment, parents have little to no understanding of acceptance and experiential avoidance, which would affect the way they comprehend the items on the AAQ - II. After treatment, parents' level of comprehension changes, thus fundamentally changing the way they interpret the measure. Other studies also found increase in acceptance following intervention in the post test scores of parents of children diagnosed with autism spectrum disorders [19-22]. Although another study by O'Brien [23] found significant change in the scores of Acceptance and Fusion Questionnaire (AFQ) from pre to post but the scores were not maintained in the follow up. Before any meaningful conclusions can be drawn about the impact of ACT interventions for parents on experiential avoidance, then, researchers need to first clarify the differences among the existing measures and improve measurement of experiential avoidance in general. Ideally, experiential avoidance would be measured behaviorally.

\section{Act and Personal Values}

The finding on personal values shows that there is a significant change across time in commitment towards health related values (Table $2 \mathrm{~b}$ ) whereas there has been no significant change found in the importance of personal values (Table 2a). Overall, valuing behaviour seems to have been impacted by treatment, at least per self - report. The differences in the commitment towards community and health have increased throughout time and phases of assessment, which might be explained as the effect of intervention and that might have also led to reduction in avoidance of the community; their commitment towards health of their children and family members increased as a result that they could see the importance and effectiveness of treatment leading to better outcomes in the both physical and psychological health in their children. Neither the Blackledge and Hayes [16] study nor the pilot by Murrell et al. [18] assessed valuing behaviour, so there is no data other than the data from this study from which to draw conclusions about the impact of ACT parenting intervention on valuing behaviour as such. Had the sample for the current study been larger, we might have seen statistically significant changes in mean PVQ scores in importance and commitment.

\section{Conclusion}

In the study done by O'Brien [23] found that there has been a significant impact of the intervention on valuing behaviour but the tools used were different from the current study and they only emphasized on the impact of ACT in the parenting domain and found there has been significant change in the scores post intervention. Though the sample size was small and long term effects of ACT could not be assessed, the findings of our study were highly consistent with the theory and philosophy behind ACT. It can, therefore, be assumed that the ACT intervention may impact removal of cognitive and affective barriers (by targeting fusion and avoidance) and therefore increase parent perceptions about their own effectiveness. However, this would depend on the existence of effective skills in the first place, and 
therefore suggest that future studies supplement ACT with behavioural skills training. There is notable absence of measures of parenting practices in the field, and future researchers should consider the development and validation of alternate parenting practices measure prior to or in conjunction with any evaluation of the effectiveness of ACT parent training.

The findings, therefore, imply that ACT may have promise in helping parents better to adjust to the difficulties in raising children diagnosed with NDD. These indicate the need for replication, more research and more intensive psychological intervention using ACT with them.

\section{References}

1. Blackledge JT, Hayes SC (2006) Using acceptance and commitment training in the support of parents of children diagnosed with autism. Child \& Family Behaviour Therapy 28(1): 1-18.

2. Branstetter RA, Cushing C, Douleh T (2009) Personal values and pain tolerance: Does a values intervention add to acceptance? J Pain 10(8): 887-892.

3. Breslau N, Davis GC (1986) Chronic stress and major depression. Arch Gen Psychiatry 43(4): 309-314.

4. Soria C, Callu D, Viguier D, El Sabbagh S, Bulteau C, et al. (2008) Parental report of cognitive difficulties, quality of lie and rehabilitaiton in children with epilepsy or treated for brain tumour. Dev Neurorehabil 11(4): 268 - 275.

5. Ciarocchi J, Blackledge JT, Hayes SC (2005) Personal Values Questionnaire. JT Blackledge.

6. Coyne LW, Wilson KG (2004) The role of cognitive fusion in impaired parenting: An RFT analysis. International Journal of Psychology and Psychological Therapy 4(3): 469- 486.

7. Deater -Deckard K (1998) Parenting stress and child adjustment: Some old hypotheses and new questions. Clinical Psychology: Science and Practice 5: 314-332.

8. DeMyer MK (1979) Parents and children in autism. Toronto, Wiley, India.

9. Gifford EV, Kohlenberg BS, Hayes SC, Antonuccio DO, Piasecki MM, et al. (2004) Acceptance based treatment for smoking cessation. Behaviour Therapy 35: 689-705.

10. Hayes SC, Bissett RT, Korn Z, Zettle RD, Rosenfarb IS, et al. (1999) The impact of acceptance versus control rationales on pain tolerance. Psychological Record 49(1): 33-47.

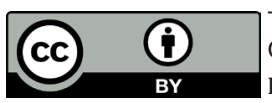

This work is licensed under Creative Commons Attribution 4.0 License

DOI:10.19080/PBSIJ.2017.3.555614
11. Hayes SC, Bissett R, Roget N, Padilla M, Kohlenberg BS, et al. (2004) The impact of acceptance and commitment training on stigmatizing attitudes and professional burnout of substance abuse counselors. Behaviour Therapy 35: 821- 836.

12. Hayes SC, Luoma J, Bond JB, Masuda A, Lillis J (2006) Acceptance and commitment therapy: Model, processes, and outcomes. Behav Res Ther 44(1): 1-25.

13. Hocking M (2010) Stress management for parents of children with autism spectrum disorder: a pilot study to evaluate the effectiveness and usability of Acceptance and commitment Therapy. The University of Queensland, Australia.

14. Konstantareas MM (1990) A psychoeducational model for working with families of autistic children. Journal of Marital Family Therapy 16(1): 59-70.

15. Micheli E (1999) A training group for parents of autistic children. International Journal of Mental Health 28(3): 100-105.

16. Murrell AR (2005) Conditioned relational responding in parents. Dissertation Abstracts International 67: 73-86.

17. Murrell AR, Schmalz J, Mitchell PR, LaBorde CT (2009) Parent action: Acceptance and commitment training for parents. Paper presented at Association for Behavior Analysis Annual Convention, Phoenix, AZ, US.

18. O'Brien KM (2011) Evaluating the Effectiveness of a Parent training Protocol Based on an Acceptance and Commitment Therapy Philosophy of Parenting.

19. Poddar, Sinha VK (2014) Effectiveness of Acceptance and Commitment Therapy on Parents of Children and Adolescents with Neurodevelopmental Disorders. Indian Journal of Developmental Disabilities 2(2): 123-132.

20. Poddar S, Sinha VK, Mukherjee U (2015) Acceptance and Commitment Therapy on Parents of Children and Adolescents with Autism Spectrum Disorders. International Journal of Educational and Psychological Researches 1(3): 221-225.

21. Poddar S, Sinha VK, Mukherjee U (2015) Challenges of Parents having Developmentally Challenged Children- An Intervention Approach using Acceptance and Commitment Therapy. J Family Med Prim Care 4(4): 604-605.

22.Wahler, R., Rowinski, K., \& Williams, K. (2008) Acceptance and mindfulness treatments for children and adolescents: A practitioner's guide. New Harbinger Press, Oakland, CA, USA.

23. Wilson KG, Murrell AR (2004) Values work in acceptance and commitment therapy: Setting a course for behavioural treatment. The Guilford Press, New York, USA, pp. 120 -150.

\begin{tabular}{l} 
Your next submission with Juniper Publishers \\
will reach you the below assets \\
- Quality Editorial service \\
- Swift Peer Review \\
- Reprints availability \\
- E-prints Service \\
- Manuscript Podcast for convenient understanding \\
- Global attainment for your research \\
- Manuscript accessibility in different formats \\
( Pdf, E-pub, Full Text, Audio) \\
- Unceasing customer service \\
Track the below URL for one-step submission \\
https://juniperpublishers.com/online-submission.php \\
\hline
\end{tabular}

